\title{
COMIDA ÉTNICA, EXOTISMO Y OTREDAD EN CHILE. EL CASO DEL RESTAURANTE "PEUMAYÉN ANCESTRAL FOOD”
}

\author{
ETHNIC FOOD, EXOTICISM AND OTHERNESS IN CHILE. THE CASE OF THE \\ RESTAURANT "PEUMAYÉN ANCESTRAL FOOD”
}

\author{
Isabel M. Aguilera Bornand ${ }^{1}$
}

\begin{abstract}
Este artículo describe e interpreta la experiencia gastronómica de los clientes del restaurante Peumayén Ancestral Food. El restaurante, ubicado en Santiago de Chile, ofrece una gastronomía inspirada en tres pueblos indígenas: Mapuche, Aymara y Rapa Nui, de modo que los comensales se acercan al consumo simbólico de otredad. Las opiniones de los clientes, recogidas a través de técnicas netnográficas, informan sobre los sentidos de aprendizaje, riesgo y autoconocimiento que cobra el consumo alimentario en contextos signados por el exotismo. El caso de Peumayén permite indagar en torno a los mecanismos de exotización/producción de una otredad consumible, así como avanzar en delimitar el papel del consumo en el marco del multiculturalismo y las relaciones interculturales.

Palabras claves: otredad, exotismo, restauración, Chile, ancestros.
\end{abstract}

This article describes and interprets the gastronomic experience of the clients of "Peumayén Ancestral Food" restaurant. This restaurant, located in Santiago the capital city of Chile, offers a cuisine inspired by three indigenous peoples: Mapuche, Aymara and Rapa Nui, so that diners approach the symbolic consumption of otherness. The opinions of the clients, collected through netnographic techniques, inform about the meanings of learning, risk and self-knowledge that food consumption acquires in contexts marked by exoticism. The case of Peumayén allows to investigate the mechanisms of exoticization / production of a consumable other, as well as to advance in delimiting the role of consumption within the frame of multiculturalism and intercultural relations.

Key words: Otherness, exoticism, restoration, Chile, ancestors.

La fascinación por la otredad, en su doble cara de deseo y rechazo, es parte de una lógica colonialista (Bhabha 1994) que ha cobrado una particular vitalidad en las sociedades signadas por el multiculturalismo neoliberal donde la arena privilegiada de acceso al otro es el consumo (Briones 2005; Comaroff y Comaroff 2011; Hale 2002; hooks 1992). De hecho, se ha estimado que en campos del consumo cultural, como el turismo y la gastronomía, los consumidores buscan de manera consciente acercarse a la otredad étnica y/o racial (Cappeliez y Johnston 2013; Karaosmanoglu 2009). Diversos estudios han demostrado, además, que la gastronomía es un espacio de producción y gestión de otredad, es decir, un campo donde las nociones de igualdad/diferencia, propio/ajeno y general/particular juegan un papel fundamental (Brayton y Millington 2011; Fischler 1995; Germann 2007; La Cecla 1995; Lu y Alan 1995). Paralelamente, si nos apegamos a la posición de Mary Douglas (1995) y consideramos la alimentación como un sistema de comunicación, podemos pensar en los alimentos como símbolos: "cosas materiales que evocan una abundancia de significados condensados y ambiguos" (Weismantel 1994:217) y, por lo tanto, como elementos susceptibles de ser significados de diferentes maneras. Por todo lo anterior, los restaurantes se presentan como un lugar propicio para observar cómo se significa el contacto con la otredad vehiculizado por el consumo alimentario.

Este artículo busca describir e interpretar, desde la perspectiva de los clientes, la experiencia de comer en Peumayén Ancestral Food, un restaurante cuya oferta simbólica es la representación de un alter indígena, esto es, los pueblos Aymara, Mapuche y Rapa Nui.

Para trabajar en torno a este objetivo se visitó el restaurante en sucesivas oportunidades, aunque sin

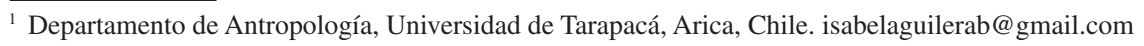


interactuar directamente con clientes. Para conocer sus narrativas se privilegió una aproximación desde la netnografía, centrada en la recopilación de opiniones desde TripAdvisor. Este material forma parte de una primera fase de un estudio que, en el futuro, debería complementarse a través de técnicas etnográficas. El análisis de opiniones y nuestras visitas sugieren que la propuesta de Peumayén pone en juego estrategias propias del exotismo alimentario (Régnier 2004) y que, desde el punto de vista de los comensales, incorporar comida ancestral vehiculiza sentidos de riesgo, aprendizaje y autoconocimiento.

\section{El Gusto por el Otro en el Marco de la Alimentación}

Para explicar la búsqueda de un contacto con la otredad a través del consumo se han puesto en juego aproximaciones diferentes. Por una parte, se vincula el gusto por el otro con la nostalgia y la búsqueda de autenticidad (Comaroff y Comaroff 2011; hooks 1992; Wang 1999; Zukin 2008); por otra parte, se ha vinculado dicho gusto con el cosmopolitismo, la diferenciación de los mercados y la emergencia de particularismos (Cappeliez y Johnston 2013; Contreras y Gracia 2005; Rössel y Schroedter 2015; Sennett 2006). Ambas aproximaciones se verifican para el ámbito de la restauración. Así, mientras que Duruz (2008), Karaosmanoglu (2009) y Swislocki (2008) asocian el gusto por lo ajeno con el consumo nostálgico, Grosglink y Ram (2013), La Cecla (1995) y Ward et al. (1999) se decantan más bien por la hipótesis del cosmopolitismo y la distinción. En cualquier caso, no cabe duda que comer otras comidas se ha estabilizado como vía de acceso a la alteridad, sobre todo cuando el consumo alimentario se enmarca en contextos turísticos como en el caso de Paumayén.

En el marco del turismo cultural las comidas operan como "una vía de acceso a otras culturas, y a la vez permiten identificarse y distanciarse, descubrir al otro y comprenderse mejor a sí mismo" (Poulain 2007:41). Aunque para La Cecla (1995) las posibilidades de encuentro con y conocimiento del otro a través del consumo alimentario en contextos turísticos son remotas, ilusorias y mediadas por la falsedad, no es menos cierto que la autenticidad es un atributo que se negocia y no una propiedad de determinados contextos (Bruner 1994; Culler 1981; Wang 1999). Desde esta perspectiva los restaurantes pueden ser tratados como una borderzone (Bruner 2005), es decir, un espacio sui generis donde comensales, meseros y cocineros acuerdan jugar un papel que asegure la percepción de autenticidad simbólica y, por lo tanto, el disfrute de la experiencia. Todavía más, aún si aceptamos que las comidas de los otros suelen ser traducidas para los comensales o acomodadas a los paladares locales, su consumo continúa siendo potencialmente transformador y la experiencia leída como auténtica (Cohen y Avieli 2004; Grosglik y Ram 2013).

Uno de los pilares de la antropología de la alimentación es comprender el hecho alimentario como un gesto íntimo ligado a las identidades individuales y sociales (Contreras y Gracia 2005; Fischler 1995). La incorporación, el acto de hacer entrar a nuestros cuerpos los alimentos es, según Fischler, un gesto trivial y portador de consecuencias irreversibles:

Incorporar un alimento es, tanto en el plano real como en el plano imaginario, incorporar todo o parte de sus propiedades: llegamos a ser lo que comemos. (...) Es al menos la representación que se construye el espíritu humano: se considera que lo incorporado modifica el estado del organismo, su naturaleza, su identidad (Fischler 1995:66).

Esta característica del consumo alimentario explica por qué comer productos y platos desconocidos puede movilizar angustia y sensación de riesgo.

La segunda característica humana que condiciona su relación con los alimentos es el omnivorismo, condición que impulsa hacia la búsqueda de novedad y que enfrenta a los comensales a la inevitable tensión entre neofilia y neobofia (Fischler 1995). Precisamente, la tensión entre verse seducido por lo nuevo y al mismo tiempo temerle, fundamenta el exotismo alimentario (Régnier 2004).

El exotismo es una relación de alteridad que descansa en la ambigüedad y, por lo tanto, el consumo de lo exótico está cruzado por fantasías, deseos, temores y contradicciones (Germann 2007; Lalvani 1995). El exotismo alimentario, como cualquier otra forma de exotismo, no es una cualidad de determinados elementos sino una construcción activa basada en el establecimiento y explicitación de una distancia geográfica, cultural y/o temporal entre los alimentos y el comensal (Hassoun y Raulin 1995; Lu y Alan 1995; Régnier 2004). La ambigüedad, donde se asienta el deseo, sumada a la distancia, logra movilizar la sensación de desconocimiento e inconmensurabilidad que consigue hacer de un elemento ajeno un peligro que seduce.

\section{Metodología}

Para conocer Paumayén se realizaron visitas al restaurante, aunque sin el objetivo de establecer una interacción directa con quienes ahí trabajan ni con los clientes. Las visitas buscaban, ante todo, apreciar de primera mano la ambientación, el tipo de atención y la propuesta gastronómica. Además, se consultaron 
las páginas web y de Facebook del restaurante. Para acercarnos a los comensales, aspecto central de este artículo, optamos por la descarga de opiniones desde TripAdvisor, una técnica propia de la netnografía (Bernard 2004). La netnografía forma parte de métodos y técnicas de investigación centradas en internet como objeto de estudio y/o como instrumento para la producción de material empírico (Ardèvol et al. 2008; Caliandro 2014) y se utiliza preferentemente para iluminar fenómenos del ámbito del consumo (Bernard 2004; Kozinets 1998; Scaraboto et al. 2012).

La descarga de opiniones es una técnica "no obstrusiva" (Hine 2011) que permite acceder de manera textual a la interpretación de un acto de consumo (Bernard 2004). Decidimos utilizar esta técnica porque la visibilidad de Peumayén es un fenómeno vinculado a internet. El restaurante ocupó durante meses el $\mathrm{N}^{\circ} 1$ en el ranking TripAdvisor de Santiago y casi la mitad de los comensales declaran haber decidido ir después de leer las críticas en la página web. Este artículo se basa en el análisis de 375 opiniones, todas ellas descargadas y leídas en idioma original. Esta información fue analizada como una narrativa (Vásquez 2012) a través de técnicas de análisis de discurso y análisis de contenido (Scaraboto et al. 2012) utilizando el programa AtlasTi.

Los métodos basados en internet han suscitado importantes debates epistemológicos, metodológicos y éticos en la antropología y la sociología (Ardèvol et al. 2008; Caliandro 2014; Hine 2011; Kozinets 1998). Uno de ellos se desarrolla en torno a la pregunta por la honestidad -en términos de identidad y de contenidode quienes utilizan las plataformas web. No hay duda que las identidades en internet pueden o no ajustarse a la vida real, sin embargo se ha demostrado que la opacidad en lugar de conducir al falseamiento permite a las personas expresarse libremente sobre sí mismos y lo que piensan (Hine 2011; Kozinets 1998). Por otro lado, no es nuestra intención comprobar la veracidad de lo dicho, sino que considerar el texto como una interpretación orientada, en gran medida, a influir en los actos de consumo de los usuarios de Tripadvisor (Scaraboto et al. 2012). Siendo esto así, hemos abordado las opiniones en tanto narrativas de una experiencia y las hemos agrupado únicamente en función de la residencia declarada que, por cierto, no puede tomarse como sinónimo de origen, ni nacionalidad. En adelante cada opinión será identificada por un número (asignado cuando fueron descargadas) y una letra $\mathrm{C}$ si la residencia declarada es Chile y X si la residencia declarada es cualquier otra.

Tripadvisor es un medio de comunicación cuyo contenido es generado por los usuarios (Ganzaroli et al. 2017; Marchiori y Cantoni 2015) y se ha posicionado como fuente de información empírica para estudios sobre turismo y restauración. Cómo se crea la imagen de un destino (Kladou y Mauragani 2015; Menezes y Gândara 2015), cómo se significa la calidad de una comida y la satisfacción (Ganzaroli et al. 2017; Pantelidis 2010; Zhang et al. 2017), cuál es el papel de las opiniones en la toma de decisiones alimentarias (Lei y Law 2015), son cuestiones que se han estudiado analizando opiniones de Tripadvisor. Desde esta perspectiva no utilizamos una fuente particularmente novedosa, sino una de probada utilidad para investigaciones que se sitúan en el cruce entre turismo, consumo y alimentación.

\section{Peumayén: Lugar de Ensueño}

"En realidad no solo era comida chilena sino que comida de pueblos originarios del norte, centro y sur de Chile, con un estilo moderno muy gourmet" (19 C).

En 2013 abrió sus puertas el restaurante Peumayén Ancestral Food, lugar de ensueño en mapudungún. Ese año fue distinguido como la mejor apertura por el Círculo de Cronistas Gastronómicos de Chile y destacado por la prensa como un "fantástico umbral hacia las raíces étnicas chilenas", que invita a soñar un "viaje en el tiempo" hasta "ese Chile aún desconocido"”: el Chile de los ancestros. El restaurante ocupa una antigua casa del barrio Bellavista en Santiago y tanto su ambientación como su propuesta gastronómica nacen de la conjunción de sofisticación y etnicidad representada por los pueblos Aymara, Mapuche y Rapa Nui. Se trata, sin duda, de una apuesta por reivindicar lo propio en un contexto, la alta cocina en Chile, que históricamente ha estado marcado por el peso del canon europeo (Aguilera 2016).

Entre los críticos de TripAdvisor, la manera más corriente de referirse a la oferta de Peumayén es usando los conceptos que pone en juego el propio restaurante: comida ancestral y comida étnica. Pero la narración pivota también sobre otros dos pares de conceptos: chileno/indígena y tradición/modernidad.

La comida ancestral se nombra como chilena o típicamente chilena y, al mismo tiempo, como prehispánica, indígena, mapuche. Se trata de dos conjuntos de conceptos que sin coincidir en términos históricos, identitarios y políticos, se unifican en el espacio soñado de Peumayén. En este sentido, las narrativas de los comensales ofrecen una representación novedosa de lo ancestral, en la medida que parece borrarse la distancia entre contingentes desmarcados que operan metonímicamente como la nación (los chilenos, imaginariamente cercanos a la blanquitud) y los contingentes marcados étnica y racialmente que ocupan el espacio de lo particular (Briones 2002). Según el discurso de que se trate, chileno e indígena pueden o no operar como términos 
opuestos, pero lo cierto es que tampoco se trata de términos intercambiables, muestra de ello es que dos opiniones refieren a las comidas indígenas como una especificidad dentro de lo nacional y lo hacen para destacar su escasa valoración. Sin embargo, la tendencia general identifica la comida ancestral como aquella que unifica lo chileno y lo indígena a través de la fusión exitosa de tradición y modernidad:"Foods of the indigenous peoples of Chile, when set in a modern interpretation, will bring out the best of both the past and the present... and create a new future" (305 X).

Por otro lado, el restaurante es percibido como único y la singularidad se erige como un tropo discursivo central en la narración de la experiencia: "Em verdade, a palavra que melhor define tal estabelecimento é DIFERENTE! Não podemos dizer que a comida é ruim, nem boa, mas somente que é diferente de tudo o que já experimentamos"(381 X).

La diferencia percibida dota de identidad al restaurante y, paralelamente, dificulta emitir juicios sobre la comida: si la comida ancestral es novedosa y única, no existe posibilidad de comparación que sustente un juicio culinario.

La magnitud de la distancia entre Peumayén y lo conocido, sumada a una experimentación placentera de esa distancia, abre camino al exotismo: "So many unique tastings I can't even remember them all! The food is pretty exotic, so I would recommend this for adventurous eaters" (240 X). En las narraciones se suceden referencias a lo raro, extraño, poco típico e incluso desagradable, conceptos que según La Cecla constituyen "la antecámara de la curiosidad y el exotismo" (La Cecla 1995:86).

\section{Tres Claves de una Experiencia de Otredad}

\section{Palabra que explica, educa y exotiza}

La experiencia Peumayén comienza con "la panera", versión gourmet e individual del pan y la mantequilla presentada en una larga tabla rectangular. Cuando la probé por primera vez estaba compuesta de una bolita de quínoa rellena con carne de llamo y una churrasca, ambos representaban el norte; dos millokines, uno de arvejas y otro de porotos, y catuto con miel, representantes del sur; y un trozo de po'e o pan de plátano de Rapa Nui. Todos estos bocados fueron presentados como panes prehispánicos del norte y sur del país. Además, se nos informó sobre las regiones representadas por cada pan y los pueblos -Aymara, Mapuche y Rapa Nui- asociados a cada uno de ellos. La presentación de la panera es un ejercicio cartográfico que anuncia el viaje metafórico que los comensales emprenderán sentados en Peumayén, un viaje donde todo merece una explicación: desde el agua hasta la más compleja de las preparaciones parecen esconder un secreto a ser develado por los meseros y meseras. A través del relato, las preparaciones son localizadas y remitidas al pasado, concretamente a un tiempo prehispánico, aunque tanto el catuto como la churrasca están hechos de trigo y nada indica que existieran antes de la llegada de este cereal desde Europa. La fuerza de la palabra poco tiene que ver con la veracidad histórica: la mención a otro tiempo abre espacios al misterio y a la distancia, define un encuentro con lo ancestral y sienta las bases del exotismo.

La orientación turística de Peumayén hacía suponer que los empleados tendrían entrenamiento en idiomas y capacidad para explicar una carta basada en productos desconocidos, sin embargo el rol de los meseros/as va mucho más allá. Por una parte actúan como culinary brokers y median entre la comida y los comensales (Cohen y Avieli 2004:772). Pero además, su "performance garantiza la otredad de los eventos [porque] transforma la historia en mito y el pasado en una escena" (Karaosmanoglu 2009:342). La palabra toma la forma de una exposición: no es reductible a la traducción lingüística, antecede las preguntas y conduce al comensal hacia una comida distante y ajena. El contenido de la exposición se repite con mínimas variaciones de mesa en mesa según un guion donde se entrecruzan referencias al lugar y tiempo de origen de productos y preparaciones, con referencias a las técnicas culinarias y sabores de cada preparación. Este uso de la palabra configura el imaginario del aula de historia: "O garçom apresenta o couvert como se fosse uma aula de história. Cada pãozinho vem de uma parte do Chile e utiliza um ingrediente especifico" (358 X), o de antropología: "The waiter explained why horse steak was eaten in Chile which made the dinner even more interesting!" (268 $\mathrm{X})$. Cuando Peumayén deviene sala de clases y los clientes toman el lugar de estudiantes, se define la posición de desconocimiento de estos últimos, aspecto central del exotismo. Paralelamente, la mesa queda servida para que la experiencia sea narrada en términos de aprendizaje y conocimiento.

El exotismo culinario supone la puesta en marcha de estrategias opuestas pero complementarias. Por un lado, es necesario acercar los elementos nuevos hacia el espacio de lo conocido para segurizar a los comensales y contrarrestar la neofobia. Por otro, es necesario marcar y aumentar la distancia entre el comensal y el producto nuevo, excitar la neofilia (Cohen y Avieli 2004; Régnier 2004). Es decir, ni tan cerca que pierda el misterio, ni tan lejos que devenga incomestible. Este es el papel que cumple la exposición que ofrecen los/as meseros/as en Peumayén. La palabra opera como un dispositivo de producción de diferencias temporales, espaciales y culturales, y modula la distancia entre comensales y 
comida para que lo ofrecido sea deseable y seguro. Volvamos a la panera para distinguir ambos sentidos de la estrategia. El contenido del plato es definido como panes prehispánicos, concepto que reúne dos ideas que producen efectos diferentes que convergen en favor del consumo. La mención al pan, familiar en varias tradiciones alimentarias, acerca los alimentos al espacio de lo conocido y seguro. A través de la analogía con el pan, preparaciones como los millokines, que no ocupan el espacio del pan en la alimentación mapuche, consiguen ser comprendidas, aprehendidas. La mención a un tiempo prehispánico, en cambio, genera una distancia temporal y cultural que envuelve al pan de misterio. El concepto panes prehispánicos consigue que la panera sea al mismo tiempo atractiva e inocua. Poco importa si las preparaciones que conforman la panera tienen o no data prehispánica porque esa categorización está sujeta a la palabra, es ella la que crea el contenido de la panera y la distancia que media entre el alimento y los comensales.

\section{Aceptar un desafío}

Comer en Peumayén se describe como la experiencia de franquear una frontera y asomarse a lo desconocido; un acto que moviliza ideas sobre el riesgo, el disgusto y la superación de temores. Si nos apegamos a la lógica del principio de incorporación, diremos que el sujeto en su totalidad es desafiado por la novedad de sabores, productos y formas de preparación. Por eso, para consumir una comida que "quizás no sea apta para quienes prefieren lo clásico" (82 C), los críticos recomiendan tener la mente abierta, deseo de experimentación y valentía. El riesgo que imaginariamente implica incorporar alimentos nuevos se manifiesta, en su grado máximo, como imposibilidad de comer: "the food is definitely not for everybody. My wife already stopped at the starters, which I kind of liked, but my main dish was too strong for me as well" (248 X).

Aunque la percepción de riesgo y la necesidad de atrevimiento son transversales a toda la experiencia gastronómica, se concentran en los dichos sobre la carne de caballo. Los comensales explican sus resistencias a la carne de caballo a través de dos líneas discursivas, una centrada en el desconocimiento y otra, en el prejuicio. La primera de ellas es común entre los críticos que declaran residencia fuera de Chile y representa una expresión literal de neofobia angustiante: "I had the horse steak which was quite tasty but couldn't psychologically prepare myself entirely to enjoy it fully like a beef steak" (246 X).

La posibilidad de comer caballo resulta conflictiva $\mathrm{y}$ aunque el influjo del exotismo sea fuerte no consigue vencer las resistencias: "initially we were reluctant to visit this restaurant on the basis of reviews that mention horse on the menu. But we decided 'when in Rome' although that philosophy did not extend as far as actually ordering the horse" (290 X). Se confirma, entonces, que las tendencias neofóbicas se presentan con fuerza entre turistas "occidentales que visitan países del Tercer Mundo percibidos como lejanos, misteriosos o peligrosos" (Cohen y Avieli 2004:759).

Entre los críticos que declaran residencia en Chile no se encuentran referencias al desconocimiento y aparece el concepto prejuicio: "Es recomendado quitarse los prejuicios para pedir platos tan deliciosos como el caballo" (75 C). ¿Cuáles son los prejuicios que deben quitarse?, ¿cuál podría ser la fuente de esos prejuicios?, en principio no podemos descartar que provengan de la falta de costumbre, de cuestionamientos morales, de la vinculación de la carne de caballo con alguna clase social en particular o de un imaginario sobre la alta cocina que excluye al caballo. Necesitaríamos investigar más profundamente y a través de otras metodologías para conocer el origen del prejuicio, pero dejaremos planteada una hipótesis al respecto.

En Peumayén el caballo se nombra y escribe cahuello. Según se nos explicó, esa sería una voz mapuche y, por lo tanto, se establece una asociación fuerte entre carne de caballo y pueblo mapuche, vinculación que por lo demás es bastante extendida. Tanto en los discursos que buscan valorar la cultura mapuche y sus particularismos, como en aquellos que los asocia a características negativas que los alejan del ethos nacional, se encuentran referencias al consumo de carne de caballo (Aguilera 2016). El caballo representaría a los Mapuche y, por lo tanto, para comprender el riesgo asociado a su consumo es necesario apreciarlo como un signo de lo indígena. La incorporación de este tipo de signos no es en absoluto inocua en un contexto, el chileno, que pivota entre la criminalización - barbarización de los mapuche y su valorización idealizada en tanto pueblo tradicional y rural (Aguilera 2016). Así, el riesgo que se encarna en la comida ancestral se relacionaría, ante todo, con aquello que los alimentos y platos representan, con las imágenes e historias que los identifican.

Comer en Peumayén supone un encuentro fugaz aunque potencialmente profundo con la otredad, un encuentro eventualmente transformador, de ahí que los comensales se vean enfrentados a sus prejuicios y narren la experiencia como un acto de arrojo.

\section{Conocer para reconocerse}

Antes de comenzar el análisis de las opiniones sabíamos que era muy probable encontrarnos con la retórica del conocer comiendo, propia del turismo cultural y gastronómico (Cohen y Avieli 2004; Germann 2007; La Cecla 1995; Poulain 2007), pero no habíamos 
previsto su extensión e importancia. El imaginario del aula y las posiciones desiguales en relación al conocimiento son, probablemente, factores que explican la preponderancia de esta retórica, pero además debemos agregar otros dos elementos decisivos: la incidencia del exotismo alimentario y el viaje metafórico que emprenden los comensales. Proponemos, por lo tanto, que el discurso del conocer comiendo no sería posible sin un trabajo previo de exotización y fetichización que convierta a determinados productos culturales en una fuerza "hacedora de mundos" (Comaroff y Comaroff 2011:51).

Los críticos declaran haber aprendido, gracias a la exposición de los meseros/as, sobre productos americanos y su localización, rasgos culturales de los pueblos indígenas, historia de América y de Chile; y haber alcanzado un encuentro profundo con Chile, el pasado y la propia identidad, gracias al viaje: "al entrar ya te sientes viajando por Chile, es volver a nuestras raíces ancestrales" (169 C). Ese encuentro tiene lugar en un momento único donde, como sugiere la opinión Time Tunnel da comida andina, pasado y presente se fusionan:

Era uma das minhas serie favoritas, a possibilidade de se viajar no tempo é sempre fascinante e se não é possível pelo menos o passado pode ser reconstituído. Eesta é a proposta do Peumayen em uma arqueologia gastronômica viva trazer aos nossos paladares os sabores, aromas e texturas dos alimentos utilizados pelo povos andinos pré hispánicos (317 X).

La posibilidad de viajar en el tiempo no es concebida de manera ingenua. La mención a la arqueología y a la reconstrucción anuncia que los comensales saben que están frente a una representación del pasado, lo cual no impide que se embarquen en un viaje imposible. Peumayén cobra, entonces, todo su sentido como lugar de ensueño y los críticos, imbuidos en la pasión del consumo, muestran un "compromiso activo en la imaginación y estimulación mediante la potencia" (Sennett 2006:133). Es tal el compromiso que no encontramos ninguna opinión que ponga en duda la autenticidad del restaurante. Al contrario, las contadas ocasiones que aparece dicho concepto en las narraciones es para afirmar que Paumayén es auténtico, lo cual confirma que una representación bien ejecutada y rigurosa, desde el punto de vista histórico, cobra sentidos de autenticidad (Bruner 1994; Culler 1981). En este sentido Peumayén se asemeja a una borderzone, es decir, un espacio donde se encuentran turistas y local performers (en nuestro caso comensales y trabajadores del restaurante), un espacio donde se crea cultura y se lleva a cabo una teatralización donde cada actor desarrolla un papel predeterminado por un periodo de tiempo definido (Bruner 2005:192). Lo que acontece en la borderzone no es una falsificación, sino una creación donde los actores participan de manera activa, es por eso que

Los intercambios culturales [que se producen en el ámbito del turismo] brindan momentos mágicos, pequeñas epifanías en las que, cruzando en efímeros encuentros las fronteras de la diferencia, vendedores y compradores por igual se sienten tocados por lo real (Comaroff y Comaroff 2011:51).

Ese es el contexto de la narración de los críticos $\mathrm{y}$, por lo tanto, el conocimiento que adquieren y el encuentro que relatan difícilmente podría interpretarse a partir de la dicotomía entre original y simulacro.

Peumayén representa, además, un espacio de autoconocimiento, una posibilidad de conocer lo que ha permanecido velado aunque sea propio: "nuestra cultura", "nuestras tradiciones ancestrales", "nuestras raíces chilenas", "[las] tradiciones culinarias de nuestros pueblos originarios". Pareciera como si a través de sus dichos los comensales expresaran el deseo de re-ligar la propia existencia con lo indígena o, al menos, a un pasado indígena. En un contexto donde el mestizo no constituye una identidad aglutinante y donde la blanquitud parece definir lo común a los nacionales (Subercaseaux 2003), la búsqueda de un re-encuentro con lo indígena - en tanto ancestro - es novedosa, aunque por otro lado confirma una retórica común para hablar de cocina chilena: aquella de las raíces cortadas (Aguilera 2016). Imaginemos por un momento que comer en Peumayén permite a los comensales incorporar la parte india de sí mismos, es decir, que la incorporación alimentaria opera como mecanismo de transformación desde adentro. ¿Qué sujeto emergería de esta especie de acto antropofágico? $\mathrm{O}$, en términos dialécticos, si la tesis es el indígena ancestralizado -epítome de tradición y espesor cultural- y la antítesis la occidentalidad globalizada y des-raizada, ¿cuál es la síntesis que emerge comiendo en Peumayén?

Sin duda, la otredad a la que se puede acceder en el restaurante es una creación hecha a partir de signos que circunscriben y fijan lo igual y lo diferente, pero ello no clausura las posibilidades de conocimiento y autoconocimiento. La experiencia con la comida ancestral abre, desde el punto de vista de los críticos, una puerta hacia la historia, el pasado, la diversidad y un sí mismo velado.

\section{Conclusiones}

Peumayén Ancestral Food no es la copia de un original indígena, sino una propuesta nueva que se 
sirve del exotismo alimentario y, por lo tanto, de la creación/explicitación de distancias temporales y geográficas entre los consumidores y aquello consumido para ofrecer una representación de la ancestralidad significada como auténtica. Una gestión cuidadosa de la palabra y la ambientación permite que los ancestros representen la otredad y que la distancia y el misterio se erijan como factores de atracción. En Peumayén el tiempo es el pasado y el espacio, el extremo norte, la Araucanía y la Isla de Pascua. Espacios alejados de la capital y signados por la presencia indígena, una presencia que aún en el marco del multiculturalismo evoca el fantasma de la inconmensurabilidad.

Para comprender el compromiso activo de los críticos de TripAdvisor con el sueño de la ancestralidad, la palabra es fundamental. La exposición que los comensales reciben crea un ambiente donde se desarrollará una experiencia que trasciende lo estrictamente culinario para devenir contacto íntimo con la otredad. Esa intimidad, sujeta al principio de incorporación, vehiculiza la sensación de riesgo asociada al consumo de lo ajeno. Un riesgo que, sin embargo, es altamente valorado porque una vez superada la tensión entre neofilia y neofobia se abre la posibilidad del conocimiento y el contacto. La aventura que supone comer en Peumayén tiene una gran recompensa final: conocer Chile y su historia, acercarse a los pueblos indígenas y, en último término, conocerse a uno mismo.

Los tres aspectos destacados -exotismo alimentario, percepción de riesgo y posibilidades de conocimiento y encuentro con el otro- son comunes en los estudios sobre restauración étnica y típica en contextos turísticos (Germann 2007; La Cecla 1995; Poulain 2007). Al respecto, hemos intentado posicionar una mirada que no reproduzca las dicotomías copia/ original, auténtico/inauténtico que poco ayudan a comprender los sentidos que para los sujetos tienen las relaciones de alteridad y que fácilmente podrían conducir a juicios de valor y críticas infundadas sobre este tipo de ejercicios gastronómicos. Como se ha establecido más arriba, el propósito de este artículo es comprender e interpretar la experiencia de los comensales y no avanzar hipótesis sobre el sentido de la acción de los creadores y trabajadores de Peumayén.

¿Cuáles son las cualidades nutritivas de la otredad?, ¿cuál es la contribución de la comida ancestral al sujeto que la consume? A partir de nuestro acercamiento pareciera que el aporte tiene relación con el espesor histórico, expresado en la ideas de raíces y tradición, y con la autopercepción como sujetos con un pasado o una parte indígena, pero ante todo, el encuentro con la otredad parece venir a llenar una falta, un espacio vacío en el sujeto. El deseo por la otredad es un deseo nostálgico (hooks 1992), un deseo orientado hacia el pasado o, si queremos, una búsqueda de algo que se ha perdido o nunca se ha tenido. El consumo de otredad refleja esa ausencia que, en nuestro caso, parece ser el vínculo con los indígenas representados como antiguos moradores de espacios lejanos aún cuando sean contemporáneos y vivan en Santiago.

Agradecimientos: La investigación que informa este artículo fue realizada gracias al financiamiento CONICYT, PAI, Concurso nacional de apoyo al retorno de investigadores/as desde el extranjero, convocatoria 2013. Proyecto folio 821320053. Agradezco a los/as evaluadores de este artículo por sus sugerentes comentarios y recomendaciones.

\section{Referencias Citadas}

Aguilera, I. 2016. De la Cocina al Estado Nación. El Ingrediente Mapuche. Icaria, Barcelona.

Ardèvol, E., A. Estalella y D. Domínguez 2008. Introducción. En La Mediación Tecnológica en la Práctica Etnográfica, editado por E. Ardèvol, A. Estalella y A. Domínguez, pp. 9-29. ANKULEGI, San Sebastián.

Bernard, Y. 2004. La Netnographie: Une nouvelle méthode d'enquête qualitative sur les communautés virtuelles de consummation. Décisions Marketing 36:49-62.

Bhabha, H. 1994. The Location of Culture. Routledge, London.

Brayton, S. y B. Millington 2011. Renovating ethnic identity on restaurant makeover. Social Identities 17:185-200.

Briones, C. 2002. Mestizaje y blanqueamiento como coordenadas de aboriginalidad y nación en Argentina. RUNA 23:61-88.
Briones, C. 2005. Formaciones de alteridad: contextos globales, procesos nacionales y provinciales. En Cartografias Argentinas: Políticas Indígenas y Formaciones Provinciales de Alteridad, editado por C. Briones, pp. 11-43. Antropofagia, Buenos Aires.

Bruner, E. 1994. Abraham Lincoln as authentic reproduction: A critique of postmodernism. American Anthropologist 96:397-415.

Bruner, E. 2005. Culture on Tour. Etnographies of Travel. The University of Chicago Press, Chicago.

Caliandro, A. 2014. Ethnography of virtual worlds, netnography, and digital ethnography. En Handbook of Business Anthropology, editado por P. Sunderland y R. Denny, pp.783-761. Left Coast Press, Walnut Creek.

Cappeliez, S. y J. Johnston 2013. From meat to "real-deal" rotis: Exploring everyday culinary cosmopolitanism. Poetics 41:433-455. 
Cohen, E. y N. Avieli 2004. Food in tourism. Attraction and impediment. Anals of Tourism Research 31:755-778.

Comaroff, J. y J. Comaroff 2011. Etnicidad S.A. Katz Editores, Madrid.

Contreras, J. y M. Gracia 2005. Alimentación y Cultura. Perspectivas Antropológicas. Ariel, Barcelona.

Culler, J.1981. Semiotics of Tourism. American Journal of Semiotics 1:127-140.

Douglas, M. 1995. Las estructuras de lo culinario. En Alimentación y Cultura. Necesidades, Gustos y Costumbres, compilado por J. Contreras, pp. 171-198. Universitat de Barcelona, Barcelona.

Duruz, J. 2008. Food as nostalgia: Eating the fifties and the sixties. Australian Historical Studies 29:231-250.

Fischler, C. 1995. El (h) Omnívoro. El Gusto, la Cocina y el Cuerpo. Anagrama, Barcelona.

Ganzaroli, A., I. De Noni y P. van Baalen 2017. Vicious advice: Analyzing the impact of TripAdvisor on the quality of restaurants as part of the cultural heritage of Venice. Tourism Management 61:501-510

Germann, J. 2007. Eating difference. The cosmopolitan mobilities of culinary tourism. Space and Culture 10:77-93.

Grosglik, R. y U. Ram 2013. Authentic, speedy and hybrid. Food, Culture \& Society 16:223-243.

Hale, Ch. 2002. Rethinking indigenous politics in the era of the indio permitido. NACLA Report on the Americas 38:16-37.

Hassoun, J.-P. y A. Raulin 1995. Homo exoticus. En Mille et Une Bouches. Cuisines et Identités Culturelles. Autrement, editado por S. Bassies, pp. 119-129 Col. Mutations/Mangeurs 154, Paris.

Hine, Ch. 2011. Internet research and unobtrusive methods. Social Reseach Update 61:1-4.

hooks, b. 1992. Black Looks: Race and Representations. South End Press, Boston.

Karaosmanoglu, D. 2009. Eating the past. Multiple spaces, multiple times - performing Ottomaness in Istambul. International Journal of Cultural Studies 12:339-358.

Kladou, S. y E. Mavragani 2015. Assessing destination image: An online marketing approach and the case of TripAdvisor. Journal of Destination, Marketing \& Management 4:187-193.

Kozinets, R. 1998. On netnography: initial reflections on consumer research investigations of cyberculture. Advances in Consumer Research 25:366-371.

La Cecla, F. 1995. Faux contact. En Mille Et Une Bouches. Cuisines et Identités Culturelles, editado por S.Bassies. Autrement, pp. 119129. Col. Mutations/Mangeurs 154, Paris.

Lalvani, S. 1995. Consuming the exotic other. Critical Studies in Mass Communication 12:263-286.

Lu, S. y G. Alan 1995. The presentation of ethnic authenticity: Chinese food as a social accomplishment. The Sociological Quarterly 3:535-553.
Lei, S. y R. Law 2015. Content analysis of TripAdvisor reviews on restaurants: A case study of Macau. Journal of Tourism 16:17-28.

Marchiori, E. y L. Cantoni 2015. The role of prior experience in the perception of a tourism destination in user generated context. Journal of Destination, Marketing \& Management 4:194-201.

Menezes, C. y J. Gândara 2015. Los discursos fotográficos de los viajeros. Curitiba-Brasil en Tripadvisor. Estudios y Perspectivas en Turismo 24:627-645.

Pantelidis, I. 2010. Electronic meal experience: a content analysis of online restaurant Comments. Cornell Hospitality Quarterly 51:483-491.

Poulain, J.P. 2007. Los patrimonios gastronómicos y sus valorizaciones turísticas. En Patrimonio Gastronómico y Turismo Cultural En El Mediterráneo, editado por J. Tresseras y X. Medina, pp. 39-71. IBERTUR, Barcelona.

Régnier, F. 2004. L' Exotisme Culinaire. Essai sur les Saveurs de l'Autre. PUF, Paris.

Rössel, J. y J. Schroedter 2015. Cosmopolitan cultural consumption: preferences and practices in a heterogenous, urban population in Switzerland. Poetics 50:80-95.

Scaraboto, D., C. Vargas y D. Costa 2012. How consumers persuade each other: rhetorical strategies of interpersonal online communities. Brazilian Administration Review 9:246-267.

Sennett, R. 2006. La Cultura del Nuevo Capitalismo. Anagrama, Barcelona.

Subercaseaux, B. 2003. La construcción de la nación y la cuestión indígena. En Nación, Estado y Cultura en América Latina, editado por A. Castillo, E. Muzzopappa, A. Salomone, B Urrejola y C. Zapata, pp. 69-80. Ediciones Facultad de Filosofía y Humanidades, Universidad de Chile, Santiago.

Swislocki, M. 2008. Culinary Nostalgia: Regional Food Culture and the Urban Experience in Shanghai. Stanford University Press, Stanford.

Vásquez, C. 2012. Narrativity and involvement in online consumer reviews. The case of TripAdvisor. Narrative Inquiry 22:105-121.

Wang, N. 1999. Rethinking authenticity in tourism experience. Annals of Tourism Research 26:349-370.

Ward, A., L. Martens y W. Olsen 1999. Consumption and the problem of variety: cultural omnivorousness, social distintion and dining out. Sociology 33:105-127.

Weismantel, M. 1994. Alimentación, Género y Pobreza en los Andes Ecuatorianos. Ediciones Abya-Yala, Ecuador.

Zhang, H., P. Ji, J. Wang y X. Chen 2017. A novel decision support model for satisfactory restaurant utilizing social information: A case study of TripAdvisor.com. Tourism Management 59:281297.

Zukin, S. 2008. Consuming authenticity. Cultural Studies 22:724-748.

\section{Nota}

${ }^{1}$ Las Últimas Noticias, Lunes 24 de Febrero de 2014. Sección Tiempo Libre. "Peumayén: el sabroso Chile olvidado", por Rodolfo Gambetti. 